\title{
Quantitative Analysis of Video Cloud Interaction Used in HFC Network
}

\author{
Zhengxiang $^{1}$, DaiYaoNan ${ }^{2}$ \\ ${ }^{1}$ Deputy Director of Chief Engineer Office, Wuhan Radio and Televison Station, No. 677 Jianshe Avenue, Wuhan, Hubei \\ Province, China \\ ${ }^{2}$ Undergraduate, Mechanical Engineering Design \& Automatics(machine-electron engineering speciality), Wuhan Institute of \\ Technology, No.693 XiongChu Sreet, Wuhan, Hubei Province, China \\ zhengxiang@pku.org.cn, $1121758919 @$ qq.com
}

\begin{abstract}
Video cloud computing is based on the concept of cloud computing technology, it is a cloud processing scheme which takes the video from "cloud" to "terminal presentation". This paper introduces the main technical characteristics of video cloud computing, the natural advantages and typical application service of video cloud computing used in video broadcasting industry, and the quantitative analysis of investment cost when video cloud computing used in 2D/3D application, as a beneficial reference for video cloud computing utilization in value-added services of the broadcasting industry.
\end{abstract}

Index Terms - Video cloud computing, bi-directional network, IP QAM technology, Quantitative analysis

\section{Introduction}

As an integration of traditional computer technology and network technology, cloud computing automatically splits an enormous program into numerous sub-programs. According to SaaS (Software \& Services), PaaS (Platform and services) and other advanced business models, using inexpensive server cluster system, cloud computing can efficiently calculate and return feedback to users. With this technology, network service provider is able to handle tens of millions or even billions pieces of information in a couple of seconds, like "supercomputer". On the other hand, cloud computing makes full use of idle resources, to provide the required computing power, storage space, software functions and information services. The typical features are: resource sharing, on-demand, elastic scheduling, service scalability, especially the "cloud" is dynamically scalable, to match the growth in scale of application and user needs.

Video cloud computing (shorten as VCC) [1] is a solution based on the concept of cloud computing, taking video as the form of terminal presentation from cloud. The idea is to gather all computing power (including display capability) in the central server, use the central computing power to encode the front-end applications and voice output, transfer them in real time through the network, and decode them in real time for terminal presentation. Here, the terminal is simplified to the functions of network, video decoding and human-computer interaction. With a single decoder chip, you can fully understand the display effect of applications. In addition, the terminal is bi-directional, capable of transfering operation control information through network in real time to the central server running application platform in cloud for application control. Because the time delay between user control and display is very small, user can hardly tell if it is the local operation or remote access. In that way, the user experience is the same as using one's own PC.

In general, VCC has the following characteristics [2][3]:

First, it breaks the performance restriction of terminals, saves the investment cost on terminals, makes terminals lightweight. As the new services of cable television develop, existing terminals become poor in performance, difficult to support complex diverse applications and large-scale value-added services. In addition, the diversity of terminal types makes the upgrade of terminals difficult, costly and long-cycle. On the contrary, based on the results of complex calculations completed in cloud, VCC can make full use of inexpensive terminals with basic Internet access, video decoding and interaction capability. Thus, service provider can utilize the existing set-top boxes, either SD STB, HD STB, smart terminals or Internet TV, to achieve the similar user experience as smart terminals. VCC helps service provider avoid upgrading terminals frequently, protect the existing investment, accelerate the application of value-added services, and increase the number of users.

Secondly, the small time delay makes the real-time application possible. In the current environment, new services and application upgrades often require online installment on terminals. Due to the diversity, nonstandard and closed source of existing terminals, the content and business migration is high workload and time-consuming. With the addition of negative influence caused by user boot time and network status, it becomes more difficult for unified deployment and quick arrival. Whereas VCC-based operating platform with the user operation response time in 200-300 ms [4] is completely open, compatible with multiple-platform service applications and multiple-terminal coverage, beneficial for rapid deployment and new service promotion.

Thirdly, the system security is manageable and controllable. The applications and contents of VCC service platform are deployed in cloud computing center, and transferred to users in the form of video stream. Because the terminals possess neither processing power nor data, the content cannot be copied or tampered by users, and the operational security and copyright protection becomes manageable and controllable. Service provider's network security is ensured, as well as partner's content copyright.

Fourthly, resource sharing and multi-screen interaction 
come true. VCC enables seamless migration and coverage among multiple terminals, plus the synchronization of data and services, thus users can access the same video cloud computing center entrance from different locations, in different networks, with different terminal devices. Access to the same video cloud computing online services at anytime from anywhere, effectively solves service provider's difficulties when the provider want to introduce new value-added services based on various types of set-top boxes, and adapt to the resolution of $\mathrm{PC}$, mobile phone, PAD and STB independently.

Fifthly, new value-added services business model is developed. Service provider wants new growth point of interest, and inevitably chooses to carry out new value-added services. However, the traditional way to carry out value-added services is the replacement of smart terminals in a wide range, causing extremely high cost, waste of existing terminals, without the guarantee that users will choose to buy value-added services after smart terminal upgrade. Therefore, when service provider takes into account the risk of return on investment, the motive to develop new value-added services is not enough. VCC services platform provides a means for omnimedia terminal coverage, cross-platform operation, which helps service provider to dig fully the value of existing terminals for display like smart terminals, without large-scale replacement of terminals, saving tedious workload and huge investment, lowering investment risk. In addition, the investment on cloud servers can be gradually adjusted based on the user amount of value-added services, which ensures service provider's revenue and return on investment.

\section{VCC Application in Broadcasting and Television Network}

VCC platform is a basic platform, on which any kind of terminals without powerful processing ability but capable of decoding and displaying video streams can access services provided by VCC through any network. When a user uses a terminal connected to VCC platform, he is virtually using a mainstream PC with rich functions, he can play games, surf, watch films and so on so forth. All existing networks which guarantees network bandwidth and low time-delay, including telecommunications, mobile, broadcasting and television networks, are able to apply VCC technology. For the time being, bi-directional broadcasting and television cable network is one of the most suitable network for VCC application [5] [6].

1) Bi-directional broadcasting and television network has steady downward path, with $38 \mathrm{Mbps}$ bandwidth occupied by one single frequency, mainly for physical signal transmission. Compared with broadband telecommunications network (average 4Mbps bandwidth), broadband telecommunications network needs exchange routing over layers, and sometimes share bandwidth with other data services. For broadcasting and television network, the full use of edge IP QAM technology can support multiple concurrent space division multiplexing video streams. Specifically, 20 concurrent video streams are enabled for 1.8Mbps H.264 encoding technology, and high-definition 2D rendering for 4Mbps encoding.

2) After the overall digitalization conversion of broadcasting and television network, digital TV set-top boxes have been popular in large-size and medium-size cities. These set-top boxes have the ability to decode MPEG-2, and a few ones have the ability to decode H.264. In order to reduce the investment cost, during the development process of 2D users with VCC, service provider can directly make use of the interactive set-top boxes as VCC terminal equipments. Generally speaking, basic functions of VCC system require little on terminal hardware (taking advantage of existing set-top box platform) and terminal software (no browser, no middleware, no transplant or maintenance workload), and users are freed from a variety of maintenance activities, including anti-virus, backup, installation, patch and so on. With VCC system, users have access to their unified service at anytime from anywhere. Thus, broadcasting and television network has naturally the two necessities of VCC: terminals and the network. Just adding the central processing node of VCC, you can easily set up a VCC services platform.

3) VCC system effectively meets the natural security requirement of digital television. First, powerful computing capabilities of cloud computing servers enhances the security of user authorization; second, cloud monitoring servers achieve real-time monitoring on end users, to prevent unauthorized users make use of legitimate authorization; third, centralized management of copyright effectively achieve cloud security; fourth, real-time protection measurements of VCC platform make it easy to monitor digital television front end, bi-directional network linkage, and terminal storage globally.

4) In broadcasting and television system, content management, cloud storage system, content distribution system (CDN), digital rights management system (DRM), content publication systems, content billing system, are a set of solution from storage to distribution, from security to billing, which can be easily transformed into media cloud, providing complete video content for VCC services.

To build a VCC system in broadcasting and television network, the following major work is required [7] [8]:

(1) The overall planning combined with VCC technology, including building bi-directional broadcasting and television network, frequency division, edge IP QAM division, feedback channel construction, "private cloud" establishment based on resources integration in the television station, comprehensive integration of existing systems, separation between users, departments, equipments and softwares, unification of service interfaces and technical standards.

(2) Choice on terminal STB with the capacities of low latency decoding, bi-directional communication, peripheral connection.

(3) VCC service platform construction with the principle of load balancing, including background management system, application runtime systems, coding systems, etc., to support BOSS, CA and other peripheral systems.

(4) Business integration platform on which applications are provided to meet user demand, not only enabling users to 
enjoy high-quality video, but also satisfying user needs for choosing freely, sharing at anytime and storing in time.

Here are applications that the VCC system based on broadcasting and television system can provide:

(a) Personal basic information processing application. Using VCC services, users can process personal information (including personal e-mail, documents, etc.) in time. In addition, users have access to unified personal information at anytime from anywhere.

(b) Game application services. Usually, some complicated games (especially 2D and 3D games, console games) require PCs with powerful processing capacity and expensive graphics card, or PS3 and other game consoles. VCC system provides video servers running those complicated games, so that users are able to conveniently play those games via inexpensive terminals.

(c) A variety of value-added services. All common value-added services currently running on ordinary set-top boxes can be provided by VCC system, without limitation by terminal STB performance.

(d) Video distribution and management applications. Especially in the background of triple play, the industry of broadcasting and television need increase data exchange with Internet and telecommunications networks gradually. Because of the immoderate openness of Internet nowadays, there are a lot of risks, such as Trojans, viruses, electronic fraud and hacking. The use of VCC system can effectively avoid those risks, while strengthen copyright protection, thus more suitable for the development requirement of the broadcasting and television industry.

\section{Construction Cost Analysis of VCC System Supporting 2D/3D Applications}

VCC provides access services on demand, optimizes system schedule, makes full use of the cloud. Now, we briefly analyze the basic construction investment for a VCC system supporting 2D/3D applications in broadcasting and television network.

In general, the investment cost of VCC consists primarily of servers, network equipments, software and so on. For convenience, we presume 10,000 subscribers (according to a fan-out ratio of $10 \%$, the actual number of concurrent streams is 1000) to carry out quantitative investment analysis.

\section{$3.12 D$ Applications}

The platform software is provided by VCC system provider, in the form of share revenue.

Third-party softwares are mainly operating systems, databases and antivirus softwares installed on servers. WINDOWS SERVER 2012 Standard Edition ( current business price is RMB 5000) as operating system, free MYSQL as database, free Kaspersky Work Space Security solutions as antivirus, make a total cost of RMB 180,000.

For IPQAM resources, 2D applications require $4 \mathrm{Mbps}$ bandwidth, so 1000 concurrent require a total bandwidth of $4000 \mathrm{Mbps}$. While a single frequency IPQAM bandwidth is $38 \mathrm{Mbps}$, the frequency number in need is 106 . At the price of RMB 1000 for a single frequency, the total cost is
RMB106,000.

In terms of small-scale interactive games, 10T storage system is sufficient. At the price of RMB 10,000 for 1T, the cost on storage system is about RMB 100,000.

Investment cost on equipment cabinets is calculated based on the price of RMB 10,000 for each 42U rackmount equipment cabinet. We usually use $1 \mathrm{U}$ rackmount equipments, so 36 servers, 3 switches and 1 firewall make a total of $40 \mathrm{U}$.

In one word, the total investment cost of a VCC system running $2 \mathrm{D}$ interactive applications for 10,000 users is approximately RMB 800,000, the average cost RMB 80 for a single user.

\subsection{Applications}

Cable TV 3D applications refer to application with HD quality running in interactive environment which require a lot of resources. Currently, a single 3D server typically supports 14 concurrent applications, so 1000 concurrent streams need 72 servers. In the assumption of each server priced RMB 10,000 , the system investment cost is RMB 720,000. Moreover, 2 management servers (including one for backup) are in need. At the price of RMB 10,000 for each one, the total investment of VCC system on servers is RMB 740,000.

For 74 servers, 3 (redundancy considered) CISCO 48-port enterprise switches are in need, with the total price no more than RMB 30,000. While a firewall supporting 1000 user licenses, such as Trend Micro Network VirusWall with 501-1000 user licenses, the cost is around RMB 40,000.

The platform software is provided by VCC system provider, in the form of share revenue.

Third-party softwares are mainly operating systems, databases and antivirus softwares installed on servers. WINDOWS SERVER 2012 Standard Edition ( current business price is RMB 5000) as operating system, free MYSQL as database, free Kaspersky Work Space Security solutions as antivirus, make a total cost of RMB 370,000.

For IPQAM resources, 3D applications require $6 \mathrm{Mbps}$ bandwidth, so 1000 concurrent require a total bandwidth of $6000 \mathrm{Mbps}$. While a single frequency IPQAM bandwidth is $38 \mathrm{Mbps}$, the frequency number in need is 158 . At the price of RMB 1000 for a single frequency, the total cost is RMB158,000.

In terms of $3 \mathrm{D}$ interactive games, 10T storage system is sufficient. At the price of RMB 10,000 for $1 \mathrm{~T}$, the cost on storage system is about RMB 100,000.

Investment cost on equipment cabinets is calculated based on the price of RMB 10,000 for each 42U rackmount equipment cabinet. We usually use $1 \mathrm{U}$ rackmount equipments, so 74 servers, 3 switches and 1 firewall make a total of $78 \mathrm{U}$, taking up 2 42U cabinets.

In one word, the total investment cost of a VCC system running $3 \mathrm{D}$ interactive applications for 10,000 users is approximately RMB 1,430,000, the average cost RMB 143 for a single user.

\subsection{D/3D mixture}

Generally, users use 2D and 3D applications at the ratio of $7: 3$. According to the above calculation method, the 
investment supporting 1,000 concurrent flows consist of:

24 2D servers, 22 3D servers, 4 management servers (2 for backup), in a total number of 48; 2 (redundancy considered) CISCO 48-port enterprise switches and 1 24-port enterprise switch as network switching equipment; WINDOWS SERVER 2012 Standard Edition as operating system, free MYSQL as database, free Kaspersky Work Space Security solutions as antivirus; 122 IPQAM resources frequency points; 10T storage systems; 2 42U rackmount equipment cabinets.

In one word, the total investment cost of a VCC system running $2 \mathrm{D} / 3 \mathrm{D}$ mixture interactive applications for 10,000 users is approximately RMB $1,000,000$, the average cost RMB 100 for a single user.

From the above three solutions, if we do not consider the cost of platform software and other expenses such as electricity, then VCC technology makes use of inexpensive server cluster to run 2D/3D service applications at low cost. The total investment cost is averaged RMB 80-150 for a single user. Specially for $2 \mathrm{D}$ applications, if we use $1.8 \mathrm{Mbps}$ coding instead of $4 \mathrm{Mbps}$, the number of IPQAM frequency resources will be greatly reduced, along with the average cost reduced further.

On the contrary, if we do not use VCC system, to support 2D/3D service applications, we need to build large-scale interactive service platform, in addition to upgrading existing set-top boxes to interactive set-top boxes, which support mainly 2D applications with the price of RMB 600 for each. Moreover, compared with VCC system, smart terminal solution also has the following problems: high cost on hardware, terminal equipment failure, susceptible to viruses, insecure for terminal equipments, network and information data, online upgrade, difficult for rapid deployment. Thus, the use of VCC system in broadcasting and television network can effectively reduce the cost of investment and maintenance, improve real-time performance and convenience, enhanced value-added service quality.

\section{Summary}

With VCC technology, broadcasting and television station can effectively set up cloud storage, cloud media asset management system and cloud services, ensure broadcast security and copyright protection, carry out value-added services easily and quickly. Meanwhile, the steady downward path and high-bandwidth provided by broadcasting and television network, the popularization of digital TV set-top boxes is a good grounding for broadcasting and television industry to carry out 2D/3D services at low cost using VCC.

\section{References}

[1] Video Cloud platform and Functions http://www.bokecc.com/serve_plat.shtml

[2] video cloud computing platform for value-added services http://www.novel-supertv.com/templates/T_yestem_products/index.asp $\mathrm{x}$ ? nodeid $=62$

[3] LVCC Lenovo virtual cloud terminal system http://wenku.baidu.com/view/cee36320ccbff121dd36834f.htm

[4] Liu Lanlan, cloud computing applications in radio and television networks are landing http://cio.chinabyte.com/283/12435783.shtml

[5] Liu Da, video cloud services: Host - radio and television network in client mode promising http://www.sarft.net/a/16154.aspx

[6] Zhang Qiang, radio and television industry, cloud computing applications and development prospects http://www.dvben.com/2010-11/04-65040.html

[7] Peng, broadcast video services to build cloud http://wenku.baidu.com/view/df9a85c34028915f804dc2a4.html

[8] Gong Wen Qiang, cloud computing applications in the broadcasting platforms and Development in http://www.gddvb.com/news/show-16295.html 